\title{
Risk Assessment for Mobile Payment Security based on Analytic Hierarchy Process Method
}

\author{
Shan Mei-jing \\ Department of Information Science and Technology, East China University of Political \\ Science and Law, Shanghai, 201620, China
}

Keywords: Mobile payment, Analytic hierarchy process, Security assessment

\begin{abstract}
In big data ages, the security is the greatest concerns of mobile payment. Comparing to the internet payment, mobile payment has many personal and difficult problems. A risk assessment indexes system was built up through Delphi method, and the weight of each index was calculated with analytic hierarchy process, then the top risk node and security value of the whole system was gotten. According to the designed index system, fuzzy comprehensive analysis method was introduced to the security evaluation of mobile payment. The experiment illustrates this index system can effectively quantify and assess the security level of mobile payment.
\end{abstract}

\section{Introduction}

Seen from the data provided by Ten pay, WeChat payment soared 332.8\% from Q4 2013 to Q4 2014.Especially, it surged 183.9\% in Q1 2014 versus Q4 2013,much higher the growth of other quarters, which was probably pushed by the rise of red envelope users in Q1 2014.

Security issues are one of the primary obstacles to the successful development of mobile payment services. Compare to the PC payment, mobile payment has a personal security features, which includes multi-terminals can access, mobile devices easy to lost and borrow, mobile devices are commonly used, combined with the traditional offline fraud, combination of convenience and safety, etc. Mobile payment service brings the masses of convenient to the consumers. At the same time, also bring bigger challenge to information and property safety work.

For mobile payment industry chain in every link of the safety concerns, possible security risks, mobile payments are currently is a hot spot in the industry and academia. For the further implementation of the CPC central committee and state council on improving the capacity of information technology (it) safe and controllable about spirit, give full play to the mobile financial's important role in the service of the people's livelihood, on January 13, 2015, the people's bank of China issued "about promoting the guidance to the healthy development of the mobile financial technology innovation".

The security of mobile payment process in addition to using the method of qualitative analysis, in order to make safety risk control, risk can be hidden trouble of safety measure, scientifically analyze data and personal information in such aspects as confidentiality, integrity, the threat, find potential safety hazard and quantitative analysis, part of the in security risk prevention, reduction, transfer, dispersion, control and resolve the target, to provide important basis to improve the safety level of mobile payment.

Methods of risk assessment. From the research status at home and abroad, the method of quantitative risk assessment can be divided into two categories: one is risk quantification method based on probability and statistics; The second is risk quantification method based on expert knowledge. For information security risk assessment method, the key research 
information system risk analysis and the quantitative evaluation method, the assessment of the risks of information system, not only need consider the risk of independent subsystems, but also risk caused by considering the interaction between subsystems.

Need to increase the risk of a mobile payment control research in the future. In fact, the current research on mobile payment risk already have some of the work[1-7]. Literature to create a matter-element model of mobile payment risk, proposed the risk assessment reference target or risk factors, some security policies or security solutions are given. Mobile payment, however, whether the real security, risk prevention and control of the assessment link is very important, but so far for quantitative research of mobile payment risk assessment are rare. Literature [8] based on bayesian networks is proposed a quantitative risk assessment model of mobile payment. In this paper, using the analytic hierarchy process (ahp) mobile payment security risk assessment model is established, implemented on the mobile payment security hidden trouble of system qualitative and quantitative analysis, and through the Delphi method based on expert assessment, analysis the influence of various factors on the overall risk, puts forward a quantitative evaluation method for mobile payment loopholes. According to the layered idea will be divided into mobile payment security hidden danger factors layer, characteristic layer and target layer. From the threat of security vulnerabilities, security, mobile communication network of the defect and lack of regulatory policy caused experts to assess the vulnerabilities, and through the calculation of weight value of various factors to evaluate its influence on the overall goals. The experimental results show that based on Delphi method and analytic hierarchy process (ahp) method of mobile payment security evaluation is effective in quantitative and on the severity of the vulnerability assessment.

\section{Risks of mobile payment security under big data background}

\section{Security threats selection criteria and evaluation indicators}

Mobile payment's main security threats including

(1) Most mobile e-commerce apps infected

Along with the further popularization of mobile payment, intelligent mobile terminal of the payment type of App downloads, including electricity, third-party payment and the bank is a malicious virus code into hard-hit, infected with the virus software version number in all shopping pay top three kind of App.

(2) Mobile payment by more than sixty percent virus will be connected to the Internet

Through mobile payment of intercepting virus detailed analysis, the biggest characteristic is shown as silent networking, delete messages, send text messages, read text, boot from the start. Among them, "" silent networking leads to cell phone users charges consumption, at the same time, through networking, virus also the private information on the user's phone, including mobile phone net silver, pay related account password content uploaded to the specified location, lead to user privacy and property risk.

(3) Qr code key channels to pay for the spread of the virus

Understand the channels of mobile payment the spread of the virus, is vital for users to guard against the virus. Virus in addition to the mobile payment, according to a report by the secondary packaging to five categories such as mobile phone online, payment application and spreading in some electronic market, the spread of BBS, qr code has become the key to the spread of the virus mobile payment channel.

Summary, found that mobile payment based on mobile terminals, as well as by the iec compared with Internet payment has the following personal and difficult problems

1) the openness of the mobile terminal, increase the attack surface system.

2) the computing power of mobile terminal is relatively weak, limiting the use of high strength and asymmetric encryption algorithm. 
3) lack of mobile terminal hardware expansibility, limiting the U shield and the wide use of digital certificates.

4) mobile terminal software function is simplified, the browser cannot support controls, like a computer browser such as password controls.

5) mobile terminal is much lower than the computer Internet network speed, especially based on GPRS and CDMA. $2 \mathrm{G}$ or $2.5 \mathrm{G}$ communication, limits the use of the application.

Therefore, the need for mobile payment security risk index system, around the five key elements in the deal - people, accounts, CARDS, equipment, and transactions, to conduct background big data system of data analysis, through the analytic hierarchy process (ahp) to calculate each node TVC, find the greatest risk of nodes, and then targeted to crack down on illegal behavior.

\section{Big data era mobile payment security mechanisms}

Mobile payment methods including SMS payment, sweep the yard, fingerprints, sound waves, payment, etc., to produce a variety of mobile payment of structured and unstructured data.

Big data for these complex form data analysis technology for mobile payment provides the opportunities of the rapid development, and to privacy and data security in the process of mobile payment puts forward a new research project.

Big data era mobile payment security mechanism is one of the traditional information security technology in the new development of the mobile Internet era of big data, through the development of new security technology, modify the original security solutions or put forward a new solution, for the big data age mobile secure payment for security technology security.

Analytic Hierarchy Process

The foundation of the Analytic Hierarchy Process(AHP) is a set of axioms that carefully delimits the scope of the problem environment. It is based on the well-defined mathematical structure of consistent matrices and their associated right eigenvector's ability to generate true or approximate weights. The AHP methodology compares criteria, or alternatives with respect to a criterion, in a natural, pair wise mode. To do so, the AHP uses a fundamental scale of absolute numbers that has been proven in practice and validated by physical and decision problems experiments.

AHP algorithm basic steps:

Step 1: Define the problem and determine the kind of knowledge sought. Structure the decision hierarchy from the top with the goal of the decision, then the objectives from a broad perspective, through the intermediate levels (criteria on which subsequent elements depend) to the lowest level (which usually is a set of the alternatives).

Step 2:Construct a set of pairwise comparison matrices. Each element in an upper level is used to compare the elements in the level immediately below with respect to it.

Use the priorities obtained from the comparisons to weight the priorities in the level immediately below. Do this for every element. Then for each element in the level below add its weight values and obtain its overall or global priority. Continues this process of weighting and adding until the final priorities of the alternatives in the bottom most level are obtained.(See Table 1) 
Table 1. Satty Nine Evaluation System

\begin{tabular}{|c|c|c|}
\hline $\begin{array}{l}\text { Intensity of } \\
\text { Importance }\end{array}$ & Definition & Explanation \\
\hline 1 & Equal importance & $B_{\text {i and }} B_{j}$ contribute equally to the objective \\
\hline 3 & Moderate importance & Experience and judgement slightly favor $B_{i}$ over $B_{j}$ \\
\hline 5 & Strong importance & Experience and judgement strongly favor $B_{i}$ over $B_{j}$ \\
\hline 7 & Very strong or demonstrate importance & $\begin{array}{l}\text { An activity is favored very strongly over } B_{i} \text {, its dominance } \\
\text { demonstrated in practice }\end{array}$ \\
\hline 9 & Extreme importance & $\begin{array}{l}\text { The evidence favoring } B_{i} \text { over } B_{j} \text { is of the highest } \\
\text { possible order of affirmation }\end{array}$ \\
\hline $2,4,6,8$ & $\begin{array}{l}\text { For compromise between the above } \\
\text { values }\end{array}$ & $\begin{array}{l}\text { Sometimes one needs to interpolate a compromise } \\
\text { judgment numerically because there is no good word to } \\
\text { describe it }\end{array}$ \\
\hline $\begin{array}{l}\text { Reciprocals } \\
\text { of above }\end{array}$ & $\begin{array}{l}\text { If activity } \mathrm{i} \text { has one of the above } \\
\text { nonzero numbers assigned to it when } \\
\text { compared with activity } \mathrm{j} \text {, then } \mathrm{j} \text { has the } \\
\text { reciprocal value when compared with } \mathrm{i} \text {. }\end{array}$ & $\begin{array}{l}\text { A comparison mandated by choosing the smaller element } \\
\text { as the unit to estimate the larger one as a multiple of that } \\
\text { unit. }\end{array}$ \\
\hline Rational & Ratios arising from the scale & $\begin{array}{l}\text { If consistency were to be forced by obtaining } n \text { numerical } \\
\text { values to span the matrix. }\end{array}$ \\
\hline $1.1-1.9$ & For tied activities & $\begin{array}{l}\text { When elements are close and nearly indistinguishable; } \\
\text { moderate is } 1.3 \text { and extreme is } 1.9\end{array}$ \\
\hline
\end{tabular}

Judgment matrix setup: values from 9 to 1 are used to show factors important level (or decision makers' preferences) from the most to the least. This scaling method is recommended by psychologists who believe that people can distinguish no more than $7(\mp 2)$ objects. Table 1 presents the definitions of different values used in judgment matrix. Usually, values in the judgment matrix are assigned by experts or acquired from surveys.

$C$ is $\mathrm{n \times n}$ matrix, has following properties:

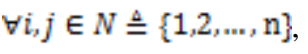

$\left\{\begin{array}{c}b_{i j}>0 \\ b_{i i}=1 \\ b_{j i}=1 / b_{i j}\end{array}\right.$ (Equation 1)

Step 3: Arrangement of relative importance of criteria: this step determines factors' weight based on the relative importance of the layer of $i+1$ to the layer of $i$ in the terms of element $j$. This calculation requires inputs from judgment matrix's maximum eigenvalue $\lambda_{m}$ and its corresponding eigenvector, which could be acquired by polymer root algorithm.

Step 4: Consistency check: Consistency index $(C I)$

$$
C I=\frac{\lambda_{m a x}-n}{n-1}(\text { Equation } 2)
$$

is used to evaluate judgment matrix's consistency. When $\lambda_{\max }=n, C I=\frac{\lambda_{\max }-n}{n-1}=0$, complete consistency is achieved, and a large CI indicates lower judgment matrix's consistency. Generally, judgment matrix is consistent when $C I<0.10$, or factors' weights needs to be reevaluated until reached new consistency. Judgment matrix's consistency has a correct this phenomenon. The corrected consistency is expressed in Equation 2.

Step 5: Global ranking: all the factors within the same

layer are assigned weights based on their relative

importance to target layer. Ranking is carried

from the highest layer to the lowest layer. Global

ranking's weighted consistency(Equation3) is checked by $C I$, and the threshold value is also 0.10 .

$C R=C I / R I \quad($ Equation 3) 
Where $\lambda_{m}$ is the maximum edge value.(See Table2 and Table3)

\begin{tabular}{|c|c|c|c|c|c|c|c|c|c|}
\hline $\begin{array}{l}\text { Dimension of } \\
\text { matrix }(n)\end{array}$ & 1 & 2 & 3 & 4 & 5 & 6 & 7 & 8 & 9 \\
\hline$R I$ & 0 & 0 & 0.58 & 0.90 & 1.12 & 1.24 & 1.32 & 1.41 & 1.45 \\
\hline
\end{tabular}

\begin{tabular}{l}
\multicolumn{9}{c}{ Table 3.Recursive calculation method of hierarchical total ordering } \\
\begin{tabular}{|l|l|l|l|l|l|}
\hline \multirow{3}{*}{ Layers } & $A_{1}$ & $A_{2}$ & $\cdots$ & $A_{m}$ & \multirow{2}{*}{ Blayer hierarchical total ordering weight } \\
\cline { 2 - 5 } & $a_{1}$ & $a_{2}$ & $\cdots$ & $a_{m}$ & \\
\hline$B_{1}$ & $c_{11}$ & $c_{12}$ & $\cdots$ & $c_{1 m}$ & $b_{1}=a_{1} c_{11}+a_{2} c_{12}+\cdots+a_{1} c_{1 m}$ \\
\hline$B_{2}$ & $c_{21}$ & $c_{22}$ & $\cdots$ & $c_{2 m}$ & $b_{2}=a_{1} c_{21}+a_{2} c_{22}+\cdots+a_{m m} c_{2 m}$ \\
\hline$\vdots$ & $\vdots$ & $\vdots$ & & $\vdots$ & $\vdots$ \\
\hline$B_{n 1}$ & $c_{n 1}$ & $c_{n 2}$ & $\cdots$ & $c_{n m}$ & $b_{n 1}=a_{1} c_{n 1}+a_{2} c_{n 2}+\cdots+a_{m m} c_{n m}$ \\
\hline
\end{tabular}
\end{tabular}

\section{Case Study}

\section{Decision goal and main factors}

From previous analysis, decision-making goal includes four criteria layers. Each criteria layer includes two or three major categories factors (Figure 1).

\section{Construct mobile payment risk}

Step1: Through identifying the security risk factors of mobile payment, construct the security risk hierarchy model, here $A_{i,}(i=1,2,3,4 ; j=1,2, \cdots$, denotes criteria layers and major categories factors separately.

Step2: According to the survey and fuzzy Delphimethod, construct hierarchyjudgment matrix $C$ and check the consistency.

$$
C=\left(\begin{array}{cccc}
1 & 2 & 3 & 5 \\
1 / 2 & 1 & 1 & 1 \\
1 / 3 & 1 & 1 & 3 \\
1 / 5 & 1 & 1 / 3 & 1
\end{array}\right) \text {, here the matrix eigenvalue is } \lambda_{\max }=4.16 \epsilon \text {, substitute it into Equation }
$$

(2) and (3), achieved $C R=0.0622<0.1$, denotes the matrix satisfies the consistency.

Step3: Arrangement of relative importance of criteria. Calculate thejudgment matrix's maximum eigenvalue's corresponding eigenvector, which could be acquired by polymer root algorithm. This step determines factors' weights based on the relative importance of elements $A_{1}, A_{2}, A_{2}, A_{4}$ in terms of the goal target layer, $u=(0.4886,0.1834,0.2129,0.1152)$

Step4: Similarly, calculate factors' weights based on the relative importance of elementsin terms of the criteria layer, achieve the following:

Paired Comparisons. Compare $B_{1}, B_{2}, B_{a}$, achieve judgment matrix

$D_{1}=\left(\begin{array}{ccc}1 & 2 & 5 \\ 1 / 2 & 1 & 3 \\ 1 / 5 & 1 / 3 & 1\end{array}\right)$, where the max eigenvalue $\lambda_{\max }=3.0037$, substitute into Equation $(2)$ and (3), achieved $C R=0.0036<0$. Indicates the judgment matrix satisfies the consistency.

The weights vector of factors $B_{1}, B_{2}, B_{\mathrm{a}}$ in terms of criteria factor is $v_{1}=(0.5813,0.3092,0.1096)$

Paired Comparisons. Compare $B_{4}, B_{5}, B_{6}$ achieve the judgment matrix 
$D_{2}=\left(\begin{array}{ccc}1 & 2 & 1 \\ 1 / 2 & 1 & 1 \\ 1 & 1 & 1\end{array}\right)$, where the max eigenvalue $\lambda_{\max }=3.0537$, substitute into Equation (2) and

(3), achieved $C R=0.0516<0.1$ Indicates the judgment matrix satisfies the consistency.

The weights vector of factors $\quad B_{4}, B_{5}, B_{6}$ in terms of criteria factor

$v_{2}=(0.4111,0.2611,0.3278)$

Paired Comparisons. Compare $B_{7} B_{8}$ achieve judgment matrix

$D_{a}=\left(\begin{array}{ll}1 & 1 \\ 1 & 1\end{array}\right)$ where themaxeigenvalue

$\lambda_{\max }=2$, substitute into Equation (2) and (3), achieved $C R=0<0.1$. Indicates the judgment matrix satisfies the consistency. The weights vector of factors

$B_{7^{x}} B_{g}$ in terms of criteria factor $A_{a}$ is $v_{a}=(0.5,0.5)$.

Paired Comparisons Compare $B_{90} B_{10}$ achieve judgment matrix

$D_{4}=\left(\begin{array}{ll}1 & 1 \\ 1 & 1\end{array}\right)$

where themaxeigenvalue $\lambda_{\max }=2$. Substitute into Equation (2) and (3), achieved $C R=0<0.1$ Indicates the judgment matrix satisfies the consistency. The weights vector of factors

$B_{9}, B_{10}$ in terms of criteria factor $A_{4}$ is $v_{4}=(0.5,0.5)$

Step 5: Global ranking. See table 4

Table 4.Mobile Payment Security Risk Hierarchy Global Ranking

\begin{tabular}{|l|l|l|l|l|l|l|}
\hline $\mathrm{B}$ & $A_{1}$ & $A_{2}$ & $A_{2}$ & $A_{4}$ & Weights & Ranking \\
\hline$B_{1}$ & 0.5813 & & & & 0.2840 & 1 \\
\hline$B_{2}$ & 0.3092 & & & & 0.1510 & 2 \\
\hline$B_{a}$ & 0.1096 & & & & 0.0535 & 8 \\
\hline$B_{4}$ & & 0.4111 & & & 0.0754 & 6 \\
\hline$B_{5}$ & & 0.2611 & & & 0.0479 & 9 \\
\hline$B_{6}$ & & 0.3278 & & & 0.0601 & 7 \\
\hline$B_{7}$ & & & 0.5 & & 0.1064 & 3 \\
\hline$B_{9}$ & & & 0.5 & & 0.1064 & 4 \\
\hline$B_{9}$ & & & & 0.5 & 0.0768 & 5 \\
\hline$B_{10}$ & & & & 0.5 & 0.0384 & 10 \\
\hline
\end{tabular}

\section{Results Analysis}

Through the above analysis and calculation show that influential factors for mobile payment security hidden danger is vulnerability of mobile terminal and third party payment platform. Therefore, in the protection of mobile payment information security and privacy protection, hardware and application of the mobile terminal security research should be strengthened, for the third party payment platform security must also be increasing. At the same time with the increase of access to mobile Internet terminals, mobile Internet business frequently occur, the safety of the wifi also need to be taken seriously. For research of weight smaller policy regulation, should be based on the principle of combining the cost and benefit consideration. 


\section{Conclusion}

In the mobile payment security risk analysis, this paper introduces analytic hierarchy process (ahp), adopted Delphi method combined with analytic hierarchy process to calculate the possible degrees, for the factors of the mobile payment security hidden danger index weight calculation, is solved based on group decision and fuzzy multi-index concentrated human judgment, for scientific research and decision-making to provide a theoretical basis and innovation in the mobile payment risk control study, for further upgrading comprehensive prevention and control system, standard mobile payment product innovation, to build secure payment environment provides a train of thought.

\section{Acknowledgements}

This work was financially supported by Humanity and Social Science Youth foundation of Ministry of Education of China(No. 14YJCZH020); National Social Sciences Program Fund (NO. 16BFX085) and China Law Society Fund (NO. CLS(2015)D073).

\section{References}

[1] Zhang Juncai, Zhao Jinhui, Qian Xun. Risk assessment of mobile payment system security based on extension theory. Proceedings of 2012 International Conference on Computer Science and Service System, 2012:880-883.

[2] Dai Hong. Risk Assessment of Mobile Payment System Security. Beijing Jiaotong University. Phd Thesis. 2010.

[3] Li Feng. Research on Security of Mobile Payment. Shandong University. Phd Thesis. 2008.

[4] Sun Wangquan. The risk analysis and safety strategy on remote mobile payment. Proceedings of 2012 IEEE Symposium on Digital Object Identifier, 2012:400-402.

[5] Wei Hongchun, Ma Ding. On Safe Solution of Mobile Payment Based on Improved 3-D Security Protocol.Computer Applications and Software. 2011, 28(4): 189-192.

[6] Xu Feng, Cui Jun, Huang Hao. Research on Security Protocol for Mobile Payment Based on J2ME. Computer Science, 2008, 35(10): 94-121.

[7] HUANG Xiao-fang, ZHOU Ya-jian, LAI Xin, YANG Yi-xian. Security Mobile Payment Scheme Based on the Third-party. Computer Engeering. 2010, 36(18): 158-162.

[8] Zhang Xuan, Lin Yifeng, Bai Chuan. Risk Assessment Model for Mobile Payment Based on Bayesian Network. Computer Engineering and Applications, 2014, 50(5): 60-64.

[9] T.L.Saaty. The Analytic Hierarchy Process. USA: McGraw Hill. 1980. 